\title{
Recognition and management of transient ischaemic attack in primary care
}

GPs play a crucial role in stroke prevention. Not only do we advise our patients and offer interventions to control vascular risk factors but we are often the first port of call for patients seeking health care after a transient ischaemic attack (TIA). ${ }^{1}$ The neurologist's definition of TIA has recently changed and patients must have had a transient clinical deficit suggesting focal brain or retinal dysfunction with a 'tissue' criterion of normal cerebral imaging. ${ }^{2}$ This will rule out stroke, which can present clinically with transient symptoms. Urgent action is needed in primary care to ensure that stroke risk is minimised after TIA.

\section{NICE GUIDANCE AND STROKE RISK}

Patients with TIA are in an accelerated phase of stroke risk, and those at highest risk of stroke within seven days of TIA can be predicted using the ABCD2 score, ${ }^{3}$ calculated using the patients' age, blood pressure in the consultation, whether speech or weakness were present, the duration of the transient event, and the presence of diabetes. The high early risk of stroke can be reduced with urgent specialist assessment and interventions, ${ }^{4}$ and consequently NICE guidance on the management of TIA in primary care recommends using the ABCD2 score to determine the urgency of specialist referral (most TIA clinics incorporate the ABCD2 score in their referral proforma), although little guidance is given on how to recognise TIA. The only intervention recommended for primary care, pending specialist confirmation of the diagnosis, is the prescription of aspirin. ${ }^{5}$ Substantial questions therefore remain about the optimal management of TIA in primary care.

\section{WHEN SHOULD WE SUSPECT TRANSIENT ISCHAEMIC ATTACK?}

There is no guidance on how we should be selecting patients with TIA from the background 'denominator' population with transient neurological symptoms. We have no reliable methods of detecting TIA at the initial consultation in primary care, a difficulty demonstrated in TIA clinic audit figures where at least $50 \%$ of patients referred from primary care do not have TIA. ${ }^{6}$ Determining who we should suspect as having had a TIA is important as the current UK care model is based on urgent

\section{"Reducing the number of referrals of patients with conditions that mimic TIA will free up scarce clinic space for those with true TIA.}

outpatient clinics, which have limited capacity. ${ }^{5}$ As the time between symptom onset and definitive intervention should be within 24 hours for patients with TIA at highest predicted stroke risk, ${ }^{5}$ delays in accessing specialist assessment should be minimal. However, this time limit for assessment is currently not met for the majority of high risk patients. ${ }^{7}$ Reducing the number of referrals of patients with conditions that mimic TIA will free up scarce clinic space for those with true TIA.

Transient neurological symptoms are common in community surveys ${ }^{8}$ and although GPs need to decide which of their patients with transient symptoms may have had a TIA, there are no prediction rules derived and validated in primary care to support accurate recognition. Although there is one specific recognition tool for TIA, ${ }^{9}$ its derivation and validation was in records of specialist assessments and requires complex calculations of nine variables; there have been no studies reported, or ongoing, testing its implementation at the first consultation in primary care.

Transient ischaemic attack clinic referral mechanisms such as proformas often specify that referrals be restricted to patients with 'focal' symptoms such as speech disturbance or unilateral weakness rather than 'non-focal' symptoms, such as confusion. However, although transient deficits in higher function such as visual neglect or apraxia are focal, they may well be interpreted as confusion by relatives and carers; a focal deficit may not always translate into a focal symptom at initial primary care presentation. Furthermore, there are many patients with transient neurological symptoms which combine both focal and non-focal features at the same time. ${ }^{8}$ The rigid application of 'focal' symptoms in referral decision making may well lead to under-detection of true TIA in the community and is another example of how the protean presentations seen in general practice do not map discretely to specialist designed assessment pathways.

\section{IS STROKE THE ONLY RISK AFTER TRANSIENT NEUROLOGICAL SYMPTOMS?}

Researchers in the Dutch TIA trial, examining the effect of different aspirin doses on stroke risk after TIA, noticed that patients with 'atypical' TIA (for example including confusion and other non-focal symptoms) had a higher cardiac event risk than patients with classical TIA (that is, with focal symptoms such as unilateral weakness). ${ }^{10}$ Furthermore, follow up of patients referred to TIA clinics shows that those 'reassured' that they have not had a TIA are in fact at higher risk of cardiac events and death than a population of patients attending hospital clinics matched for cardiovascular risk factors but who have not had an episode of transient neurological symptoms. ${ }^{6}$ A community-based cohort study also found that patients with transient neurological symptoms that were again atypical for TIA lthey had mixed focal and non-focal symptoms) had a higher risk of cardiovascular death, cardiac disease, and dementia than patients with purely focal transient neurological events that are more typical for TIA. ${ }^{8}$

Therefore, within the population of patients with transient neurological symptoms, there appear to be patients at high stroke risk and other patients at high cardiac risk and the latter may not be best served by systems that only seek to identify and treat the patients at high stroke risk. Given the substantial non-stroke vascular disease burden in patients who have had transient neurological symptoms that are not sufficiently 'focal' to warrant a TIA diagnosis, the approach of the generalist should include a full vascular risk factor assessment, rather than reassurance that their event 'was not a TIA'. Ultimately, better recognition of these different patient 


\section{“... within the population of patients with transient neurological symptoms, there appear to be patients at high stroke risk and other patients at high cardiac risk and the latter may not be best served by systems that only seek to identify and treat the patients at high stroke risk.}

populations should come from prediction rules, but there is often a reduction in the usefulness of prediction rules that have been derived and validated in specialist settings when they are translated to the generalist community setting. ${ }^{11}$ Prediction rules are needed that are derived and validated in primary care.

\section{IMPLEMENTING THE EVIDENCE FOR URGENT RISK FACTOR CONTROL}

Even if we can develop more appropriate selection of patients for urgent clinics whose specific aim is to reduce stroke risk the current NICE guidance recommends only prescribing aspirin at the initial primary care consultation, ${ }^{5}$ and patients need to wait until a specialist assessment for full vascular risk reducing medication; the majority of patients with TIA do not have critical carotid stenosis and are managed medically. ${ }^{4}$ Although there is strong evidence that urgent risk factor control in patients with a confirmed diagnosis of TIA prevents stroke, ${ }^{4}$ we do not know the net benefit of prescribing such intensive treatment regimens at an initial consultation in primary care to the larger population of patients with suspected TIA. Currently the harms and benefits of prescribing these familiar treatments lantiplatelet combinations, antihypertensive agents, and statins) in advance of a subsequent specialist assessment have not been quantified. NICE has not considered this option in its decision model of care pathways or included it in research recommendations. ${ }^{5}$

\section{CONCLUSION}

Reducing the burden of cerebrovascular disease needs primary care, but primary care in turn needs to sharpen its detection of the patient at risk and ensure that maximal protection from evidence-based therapies is initiated as soon as possible. We also need to recognise that in our pursuit of the patient with accelerated risk of stroke, we also capture patients at risk of cardiac disease. The evidence gaps of who we detect, and when we protect, remain to be closed.

\section{Daniel Lasserson,}

Senior Clinical Researcher and GP, University of Oxford, Department of Primary Care Health Sciences, New Radcliffe House, Oxford.

\section{Provenance}

Commissioned; not externally peer reviewed.

\section{Competing interests}

The author has declared no competing interests.

DOI: 10.3399/bjgp13X661011

\section{ADDRESS FOR CORRESPONDENCE}

\section{Daniel Lasserson}

University of Oxford, Department of Primary Care Health Sciences, New Radcliffe House, Radcliffe Observatory Quarter, Woodstock Road, Oxford, OX2 6GG, UK.

E-mail: daniel.lassersonđaphc.ox.ac.uk

\section{REFERENCES}

1. Lasserson DS, Chandratheva A, Giles MF, et al. Influence of general practice opening hours on delay in seeking medical attention after transient ischaemic attack (TIA) and minor stroke: prospective population based study. BMJ 2008; 337: a1569.

2. Easton JD, Saver JL, Albers GW, et al. Definition and evaluation of transient ischemic attack: a scientific statement for healthcare professionals from the American Heart Association/American Stroke Association Stroke Council; Council on Cardiovascular Surgery and Anesthesia; Council on

Cardiovascular Radiology and Intervention; Council on Cardiovascular Nursing; and the Interdisciplinary Council on Peripheral Vascular Disease. The American Academy of Neurology affirms the value of this statement as an educational tool for neurologists. Stroke 2009; 40(6): 2276-2293.

3. Johnston SC, Rothwell PM, Nguyen-Huynh $\mathrm{MN}$, et al. Validation and refinement of scores to predict very early stroke risk after transient ischaemic attack. Lancet 2007; 369(9558): 283-292.

4. Rothwell PM, Giles MF, Chandratheva A, et al. Effect of urgent treatment of transient ischaemic attack and minor stroke on early recurrent stroke (EXPRESS study): a prospective population-based sequential comparison. Lancet 2007; 370(9596): 14321442.

5. National Collaborating Centre for Chronic Conditions. Stroke: national clinical guideline for diagnosis and initial management of acute stroke and transient ischaemic attack (TIA). London: Royal College of Physicians, 2008.

6. Cameron AC, Dawson J, Quinn TJ, et al. Long-term outcome following attendance at a transient ischemic attack clinic. Int J Strok 2011; 6(4): 306-311.

7. Intercollegiate Stroke Working Party. National sentinel stroke audit. London: Royal College of Physicians, 2010

8. Bos MJ van Rijn MJ, Witteman JC, et al. Incidence and prognosis of transient neurological attacks. JAMA 2007; 298(24): 2877-2885.

9. Dawson J, Lamb KE, Quinn TJ, et al. A recognition tool for transient ischaemic attack. QJM 2009; 102(1): 43-49.

10. Koudstaal PJ, Algra A, Pop GA, et al. Risk of cardiac events in atypical transient ischaemic attack or minor stroke. The Dutch TIA Study Group. Lancet 1992; 340(8820): 630-633.

11. Moons KG, Altman DG, Vergouwe $Y$, Royston P. Prognosis and prognostic research: application and impact of prognostic models in clinical practice. BMJ 2009; 338: b606. 